\title{
Study on Active Suspension Control to Improve Vehicle Handling Stability
}

\author{
Wang Jinxiang \\ School of Mechanical Engineering \\ Southeast University \\ Nanjing, P.R. China \\ wangjx@seu.edu.cn
}

\author{
Chen Nan \\ School of Mechanical Engineering \\ Southeast University \\ Nanjing, P.R. China \\ nchen@seu.edu.cn
}

\author{
Yin Guodong \\ School of Mechanical Engineering \\ Southeast University \\ Nanjing, P.R. China \\ ygd@seu.edu.cn
}

\begin{abstract}
The effect on handling stability by active suspension is analyzed to select the most suitable modes of control input. After that, based on fuzzy sliding mode control algorithm, an active suspension controller is designed for improving performance of vehicle handling stability. Simulation is made to verify the validity of the proposed controller. Results show that the proposed controller can enhance the handling performance of the vehicle in critical maneuvering.
\end{abstract}

Keywords-Active Suspension; vehicle stability control; fuzzy sliding mode

\section{INTRODUCTION}

Active suspension control not only can provide good vibration isolation for the sprung mass to improve pitch, roll and heave response of the vehicle body, but also can achieve better handling stability in critical maneuvering.

If the tire slip angle is fixed, total lateral tire force of the axle will be reduced while the vertical load transfer between the left and right tire is increased [1]. It can be concluded in [2] that increasing front bias of load transfer distribution will lead to more understeer, on the other hand, increasing rear bias will lead to more oversteer.

It was suggested in [3] that by reducing outer front and inner rear tire load simultaneously, understeering vehicle can be corrected. And it was suggested by March [4] that a single suspension force activated on inner rear tire can lead to unfavorable roll angle. And in [5], Wang has suggested that passive suspension and active suspension for increasing ride comfort have little effect on road holding ability. In literatures, these conclusions were almost deduced formalistically with simplified linear vehicle model.

In this paper, the most suitable modes of control input for active suspension to improve handling stability are selected by simulation with a full vehicle model. Then an active suspension controller is proposed based on fuzzy sliding mode control. A simulation with maneuver of double lane change is designed to verify the effectiveness of the proposed controller.

\section{SELECTION MODES OF CONTROL FORCES INPUT}

Both the yaw rate and attitude of vehicle can be influenced by active suspension control. While in some given conditions, the influence to attitude of the sprung mass can be minimized.

This work has been supported by National Natural Science Foundation of China (Grant No. 51205058, 51105074, 50975047).
Simulations are designed to analyze the effect of different active suspension control modes on the performance of vehicle handling stability as well as vehicle vertical, pitch and roll motion.

Assume that the vector of control forces of active suspension is denoted by

$$
F_{c}=\left[F_{f l_{-} c}, F_{f r_{-} c}, F_{r l_{-} c}, F_{r r_{-} c}\right]^{T}
$$

Where $f l, f r, r l, r r$ denote front left, front right, rear left and rear right tire respectively.

\section{A. Differential control forces on front axle}

Equal and opposite control forces are applied on outer front and inner front tires respectively, with positive force on outer and negative force on inner front tire, to study the effect on cornering performance.

A 14-DOF full vehicle model is used for simulation. The vehicle model comprises the translational and rotational degrees of freedom of the sprung mass, as well as the vertical and rotational dynamics of the tire. More details of the vehicle model can be found in [6].

The simulation is conducted in Matlab/Simulink, with zero road input, tire-road adhesion being 0.9 , and initial vehicle velocity being $28 \mathrm{~m} \cdot \mathrm{s}^{-1}$. A step steering input is applied on front wheel, till both yaw rate and lateral acceleration is in steady state. The steady state lateral acceleration is denoted by $a_{y s s}$. At this moment, the positive control force $F_{f l_{c} c}=300 \mathrm{~N}$ and negative control force $F_{f r}=-300 \mathrm{~N}$ are applied on outer and inner front tires respectively. After a while the yaw rate and lateral acceleration will be resumed to steady state.

Deviations of the steady state yaw rate, lateral acceleration and roll angle between with and without the action of differential suspension forces are calculated. Then the simulation is repeated with increased step steering angle, and the deviations in different $a_{y s s}$ are achieved. As shown in Fig.1, the simulation results can be analyzed as follows: from Fig. 1, it can be observed that differential control forces applied on front axle can remarkably reduce the yaw rate and lateral acceleration, with ability to correct oversteer and to decrease the absolute value of roll angle, as the sign of roll angle is negative. When $a_{y s s}$ is smaller than $3.7 \mathrm{~m} \cdot \mathrm{s}^{-2}$, the differential suspension forces can reduce the roll angle, but has little 
effect on yaw rate and lateral acceleration; when $a_{y s s}$ falls into the region $[5.5,6.3] \mathrm{m} \cdot \mathrm{s}^{-2}$, the effect is most significant. But when $a_{y s s}$ is bigger than $6.3 \mathrm{~m} \cdot \mathrm{s}^{-2}$ the effect declines promptly.

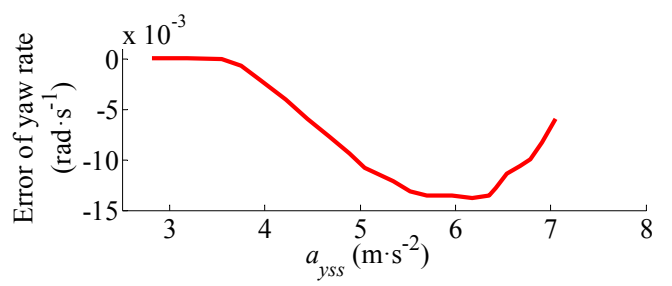

(a) Yaw rate

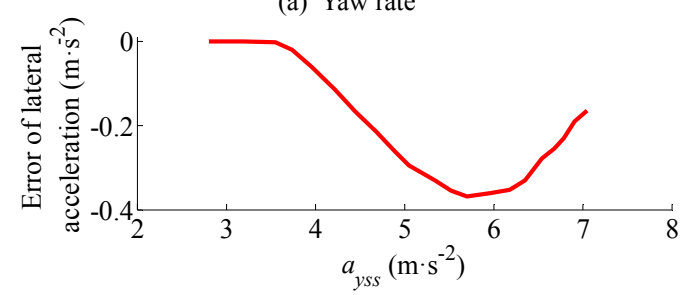

(b) Lateral acceleration

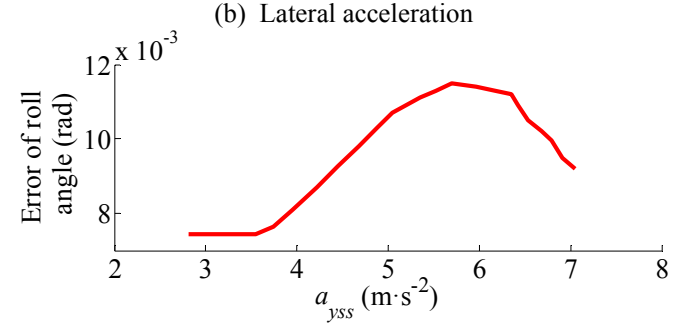

(c) Roll angle

Figure 1. Control effect when differential forces applied on front tires

\section{B. Negative control forces on outer front and inner rear tire}

Simulation condition and step steering input are identical as previously. As both yaw rate and lateral acceleration are in steady state after the step steering input, equal negative control forces are applied on outer front and inner rear tires, with $F_{f l c}=-300 \mathrm{~N}$ and $F_{r r}=-300 \mathrm{~N}$. The simulation results can be analyzed as follows: it can be observed from Fig. 2 that equal negative control forces on tires of across corners can remarkably increase the yaw rate and lateral acceleration, to be able to correct understeer whereas the absolute value of roll angle is hardly increased. When $a_{y s s}$ is smaller than $3.5 \mathrm{~m} \cdot \mathrm{s}^{-2}$, the control forces have little effect on yaw rate, lateral acceleration and roll angle; when $a_{y s s}$ falls into the region [4.7, $6.5] \mathrm{m} \cdot \mathrm{s}^{-2}$, the effect is most significant. But when $a_{y s s}$ is bigger than $6.5 \mathrm{~m} \cdot \mathrm{s}^{-2}$, the control effect on understeer correction declines promptly.

\section{Other modes of control forces input}

With negative control force acted on outer front tire and equal opposite control force on inner front tire, compared with equal negative control forces on outer front and inner rear tires, the effect on yaw rate is quite similar, but the former causes bigger roll angle increase, with almost twice roll angle error more than the latter at $a_{y s s}=4.8 \mathrm{~m} \cdot \mathrm{s}^{-2}$. Obviously, the latter mode of control forces input is better.

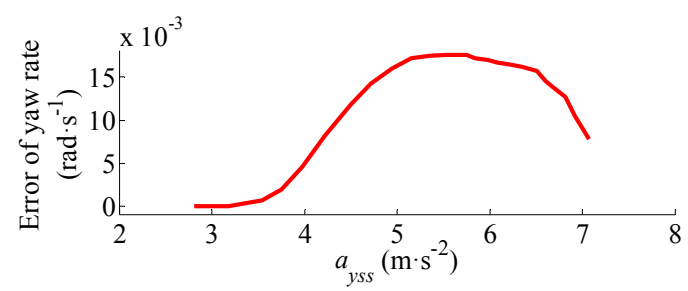

(a) Yaw rate

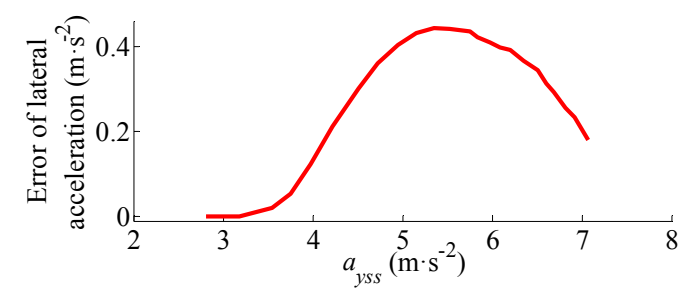

(b) Lateral acceleration

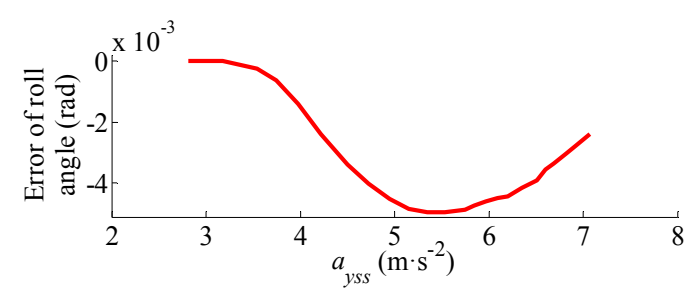

(c) Roll angle

Figure 2. Control effect when negative forces applied on diagonal tires

With equal positive control forces on outer front and inner rear tires, compared with positive control force applied on outer and equal negative control force on inner front tire, the effect on yaw rate is also similar, but the latter causes more roll angle decrease.

In summary, taking account to both riding comfort and handling stability, the following control modes of active suspension are optimal: to correct understeer, it is better to apply equal negative control forces on outer front and inner rear tires; to correct oversteer, it is better to apply positive force on outer and negative force on inner front tire.

\section{CONTROL ALGORITHM}

In this section a fuzzy sliding mode control algorithm is introduced to realize the active suspension control.

The aims of the proposed active suspension controller are tracking the desired yaw rate and improving handling stability in region of medium lateral acceleration, at the same time minimizing the adverse effect on rolling and pitching dynamics.

Based on the method of vehicle stability control in [7] and the design rules of fuzzy sliding mode control, the combined tracking error of yaw rate and side slip angle can be defined as,

$$
e=\left(r-r_{t g}+W_{\beta} \beta\right) \sin \left[\frac{\pi}{2} s a t\left(\frac{r_{t g}}{\varepsilon}\right)\right]
$$

Where, 


$$
\begin{gathered}
\operatorname{sat}(x)=\left\{\begin{array}{cc}
-1, & x \leq-1 \\
x, & -1<x<1 \\
1, & x \geq 1
\end{array}\right. \\
W_{\beta}=\left\{\begin{array}{cc}
0, & |\beta|<0.5 \beta_{\text {lim }} \\
\left(\frac{|\beta|-0.5 \beta_{\text {lim }}}{0.6 \beta_{\text {lim }}}\right)^{3}, & 0.4 \beta_{\text {lim }} \leq|\beta|<1.1 \beta_{\text {lim }} \\
1, & |\beta| \geq 1.1 \beta_{\text {lim }}
\end{array}\right.
\end{gathered}
$$

In (2), $r$ and $r_{t g}$ are real and target yaw rate respectively. Equation (2) is doubly differentiable because of the sine function. $\varepsilon$ denotes the steady state tracking error of yaw rate, as the boundary layer density of the sliding mode control. $\beta_{\text {lim }}$ denoting upper bound of the side slip angle maintaining handling stability, can be calculated empirically as,

$$
\beta_{\lim }=0.02 \mu \mathrm{g}
$$

Where $\mu$ is tire-road adhesion coefficient.

The presented control idea can be divided into several steps. Firstly with the method of fuzzy sliding mode control, a normalized control force is determined by the tracking error $e$. Then direction of the corrective yaw moment is determined with tracking error and the steering desire of the driver. After that, based on the direction, the action mode of the control forces for active suspension is decided.

The switching function is defined as,

$$
s=\lambda \dot{e}+e
$$

Inputs of the fuzzy sliding mode controller are defined as $k_{1} s$ and $k_{2} \dot{s}$, where $k_{1}$ and $k_{2}$ are scale coefficients. The output of the fuzzy sliding mode controller are normalized as control force $F_{\mathrm{ASH}}$, where the subscript $\mathrm{ASH}$ represents active suspension control for handling stability. The domain of the input and outputs are all in region $[-1,1]$.

The fuzzy sets of the inputs and output respectively are,

$$
\begin{array}{r}
\left\{k_{1} s, k_{2} \dot{s}\right\}=\{\mathrm{NB}, \mathrm{NM}, \mathrm{NS}, \mathrm{ZO}, \mathrm{PS}, \mathrm{PM}, \mathrm{PB}\} \\
F_{\mathrm{ASH}}=\{\mathrm{NB}, \mathrm{NM}, \mathrm{NS}, \mathrm{ZO}, \mathrm{PS}, \mathrm{PM}, \mathrm{PB}\}
\end{array}
$$

The membership functions are shown in Fig. 3. Lyapunov's stability theorem is applied to design the fuzzy inference rules. Based on the theorem, if the inequality $k_{1} k_{2} s \dot{s}<0$ is satisfied when (1) is not zero, then the control system is asymptotically stable. The inference rules are deduced as shown in Table 1, where the Mamdani fuzzy inference system is used, e.g., the inference rules for the first item of Table 1 can be explained as,

$$
\text { If } k_{1} s_{3} \text { is } \mathrm{NB} \text { and } k_{2} \dot{s} \text { is } \mathrm{PB} \text {, then } F_{\mathrm{ASH}} \text { is } \mathrm{ZO}
$$

The centroid method is introduced to realize defuzzification of the output.
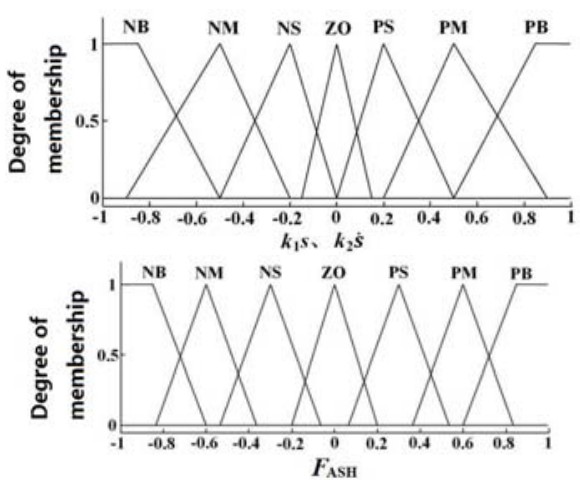

Figure 3. Membership function of the fuzzy sliding mode control

TABLE I. INFERENCE RULES OF THE FUZZY SLIDING MODE CONTROL

\begin{tabular}{|c|c|c|c|c|c|c|c|}
\hline & & & & & & \\
NB & NM & NS & ZO & PS & PM & PB \\
\hline PB & ZO & PS & PM & PB & PB & PB & PB \\
\hline PM & NS & ZO & PS & PM & PB & PB & PB \\
\hline PS & NM & NS & ZO & PS & PM & PB & PB \\
\hline ZO & NB & NM & NS & ZO & PS & PM & PB \\
\hline NS & NB & NB & NM & NS & ZO & PS & PM \\
\hline NM & NB & NB & NB & NM & NS & ZO & PS \\
\hline NB & NB & NB & NB & NB & NM & NS & ZO \\
\hline
\end{tabular}

After the normalized control force $F_{\mathrm{ASH}}$ is calculated, the desired vector of control forces can be determined based on the conclusions acquired in last section as,

$$
\begin{aligned}
\boldsymbol{F}_{d e s}= & -0.5\left[\boldsymbol{c}_{1}+\boldsymbol{c}_{2} \operatorname{sgn}\left(r_{t g}\right)\right]\left|\operatorname{sgn}\left(r_{t g}\right)\right| \widehat{\operatorname{sat}}\left(-F_{\mathrm{ASH}}\right)+ \\
& \boldsymbol{c}_{3} \operatorname{sgn}\left(r_{t g}\right) \widehat{\operatorname{sat}}\left(F_{\mathrm{ASH}}\right)
\end{aligned}
$$

Where the const vectors $\boldsymbol{c}_{1}=[1,1,1,1]^{T}, \boldsymbol{c}_{2}=[1,-1,-1,1]^{T}$, $\boldsymbol{c}_{3}=[1,-1,0,0]^{T}$, and the saturation function is defined as,

$$
\widehat{\operatorname{sat}}(x)=\left\{\begin{array}{rr}
0, & x \leq 0 \\
x, & 0<x<1 \\
1, & x \geq 1
\end{array}\right.
$$

When $F_{\mathrm{ASH}}<0$, first item of (7) is not zero, yaw moment with the same direction as $r_{t g}$ is needed, i.e. negative control forces are demanded on outer front and inner rear tires. If $r_{t g}>0$, the negative control forces are demanded on front left and rear right tires, else if $r_{t g}<0$ the forces are demanded on front right and rear left tires.

When $F_{\mathrm{ASH}}>0$, last item of (7) is not zero, yaw moment with opposite direction as $r_{t g}$ is needed, i.e., equal and opposite control forces are demanded on outer and inner front tires. If $r_{t g}>0$, the positive control force is demanded on front right tire and negative one on front left tire, else if $r_{t g}<0$, negative control force is demanded on front right tire and positive one on front left tire. 
Afterwards, considering the effect of the control forces on vehicle handling stability, the operation region of the active suspension control can be define according to the lateral acceleration as,

$$
R_{\mathrm{ASH}}=\left[1-\widehat{\operatorname{sat}}\left(\frac{c_{4}}{\mu g}\left(\left|a_{y}\right|-c_{5} \mu g\right)\right)\right] \widehat{\operatorname{sat}}\left(\frac{c_{6}}{\mu g}\left(\left|a_{y}\right|-c_{7} \mu g\right)\right)
$$

Where const coefficients $c_{4}, c_{5}, c_{6}, c_{7}$ can be determined as $c_{4}=12.5, c_{5}=0.85, c_{6}=20, c_{7}=0.35$.

\section{Simulation AND Results}

In order to simulate critical condition, a non-standard double lane change maneuver [8] is employed, an open loop front wheel steering is applied with the maximum amplitude of steering angle is $0.087 \mathrm{rad}$, as shown in Fig. 4. The 14-DOF full vehicle model is used for simulation, with zero road input. The initial velocity is $22 \mathrm{~m} \cdot \mathrm{s}^{-1}$, tire-road adhesion coefficient is 0.9 .

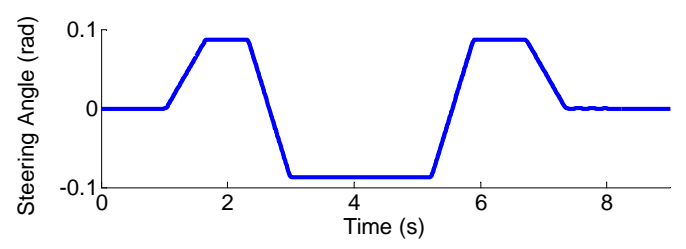

Figure 4. Front wheel steering angle

Fig. 5 shows the time histories of the yaw rate, sideslip angle and trajectory. It is observed from Fig. 5(c) that the passive vehicle, without control, cannot make the double lane change, while the vehicle with active suspension control can successfully achieve double lane change. It is shown in Fig. 5(a) that error of yaw rate for passive vehicle becomes large after $5.0 \mathrm{~s}$, but the yaw rate error of vehicle with control is smaller provided with preferable capability of yaw rate tracking. It can be seen in Fig. 5(b) that the maximum sideslip angle of passive vehicle exceeds $0.22 \mathrm{rad}$ at $5.3 \mathrm{~s}$, which means the vehicle will become unstable and slip out of the desired trajectory, but the maximum sideslip angle of the controlled vehicle is limited to less than $0.12 \mathrm{rad}$, revealing that the vehicle is stable. In Fig. 5(d), peak of the roll angle is restricted bellow $0.1 \mathrm{rad}$, similar to the passive vehicle. That is, the adverse effect of the active suspension control on roll angle is diminished.

\section{CONCLUSIONS}

In this paper, an active suspension control for improving vehicle handling stability is proposed based on fuzzy sliding mode control. The simulation results show that, compared with the passive vehicle, the controlled vehicle has more capability of handling stability in critical maneuvering. The results also show that, the proposed modes of control forces input can provided with better performance of tracking handling parameters and minimum unfavorable effects on attitude of the sprung mass.
Other means of improving vehicle handling stability such as active front wheel control (AFS), electronic stability control (ESC) are not included in this paper. The integration of these controllers with active suspension control will be considered in future study.

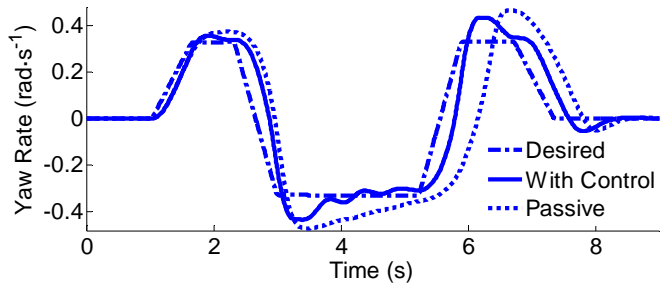

(a) Yaw rate

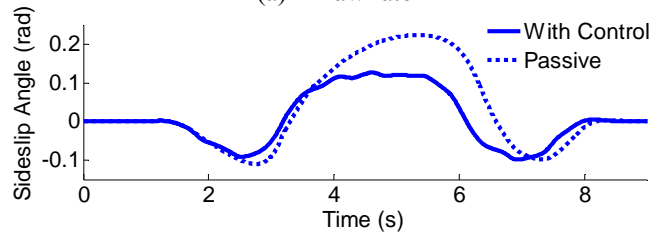

(b) Sideslip angle

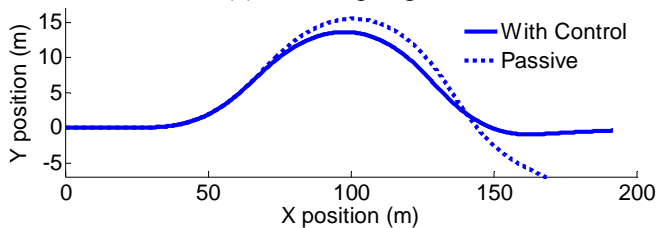

(c) Vehicle trajectory

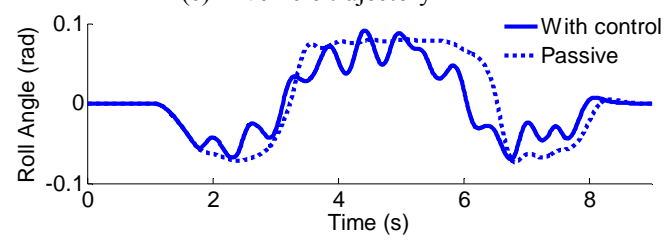

(d) Roll angle

Figure 5. Simulation results in double lane change

\section{REFERENCES}

[1] T. D. Gillespie. Fundamentals of vehicle dynamics [M]. Society of Automotive Engineers Inc, 2000.

[2] C. L. Clover, and Bernard J. E. The influence of lateral load transfer distribution on directional response [C]. SAE paper No. 930763, 1993.

[3] T. Shim, and Margolis D. Dynamic normal force control for vehicle stability enhancement [J]. International Journal of Vehicle Autonomous System, 2005, 3(1): 1-14.

[4] C. March, and T. Shim. Integrated control of suspension and front steering to enhance vehicle handling $[\mathrm{J}]$. Proceedings of the Institution of Mechanical Engineers, Part D: Journal of Automobile Engineering, 2007, 221 (D4):377-391.

[5] J. Wang, D. A. Wilson, W. Xu, and D. A. Crolla. Integrated vehicle ride and steady-state handling control via active suspensions[J]. International Journal of Vehicle Design, 2006, 42(3-4): 306-327.

[6] J. X. Wang. Study on Multi-Agent Based Integrated Vehicle Chassis Control System [D]. Ph.D. dissertation, Southeast Univ. Nanjing, China, 2010.(in Chinese)

[7] R. Rajamani. Vehicle Dynamics and Control [M]. Springer, 2006, $224-$ 239.

[8] T. H. Hwang, K. Park, S. J. Heo, S. H. Lee, and J. C. Lee. Design of integrated chassis control logics for AFS and ESP [J]. International Journal of Automotive Technology, 2008, 9(1):17-27. 